\section{$\leqslant$ Research Square}

\title{
Selective Terminal Functionalization of Linear Alkanes
}

llan Marek ( $\nabla$ chilanm@technion.ac.il )

The Technion - Israel Institute of Technology

Jeffrey Bruffaerts

The Technion - Israel Institute of Technology

Keren Buhnik-Rosenblau

The Technion - Israel Institute of Technology

Anthony Cohen

The Technion - Israel Institute of Technology

Inbar Kesten

The Technion - Israel Institute of Technology

\section{Morgan Cormier}

The Technion - Israel Institute of Technology

Yuanfei Zhang

The Technion - Israel Institute of Technology

\section{Guo-Ming Ho}

The Technion - Israel Institute of Technology

Itai Massad

The Technion - Israel Institute of Technology

\section{Nurit Edri}

The Technion - Israel Institute of Technology

\section{Hila Halfon-Verner}

The Technion - Israel Institute of Technology

\section{Fabian Glaser}

The Technion - Israel Institute of Technology

\section{Guy Horev}

The Technion - Israel Institute of Technology

Yechezkel Kashi

The Technion - Israel Institute of Technology https://orcid.org/0000-0001-8010-0068

https://orcid.org/0000-0002-2469-3345 
Keywords: linear alkanes, primary C-H bonds, biocatalytic dehydrogenation, remote hydrofunctionalization

Posted Date: January 29th, 2021

DOI: https://doi.org/10.21203/rs.3.rs-133031/v1

License: (c) (1) This work is licensed under a Creative Commons Attribution 4.0 International License. Read Full License 


\section{Abstract}

Selective undirected functionalization of strong primary $\mathrm{C}-\mathrm{H}$ bonds of linear alkanes, that do not possess directing groups, historically stands as one of the most challenging transformation in chemistry. In this Article, we report a two-step sequential strategy involving a biocatalytic dehydrogenation / remote hydrofunctionalization, as a unified and versatile approach to selectively convert linear alkanes into a large array of valuable functionalized aliphatic derivatives. The dehydrogenation is carried out by a mutant strain of Rhodococcus and the produced alkenes are subsequently engaged in a remote functionalization through a metal-catalyzed hydrometalation/migration sequence that subsequently react with a large variety of electrophiles. The judicious implementation of this combined biocatalytic and organometallic approach enabled us to develop a high-yielding protocol to site-selectively functionalize unreactive primary $\mathrm{C}-\mathrm{H}$ bonds.

\section{Main Text}

Selective functionalization of unreactive $\mathrm{C}-\mathrm{H}$ bonds represents a significant paradigm shift from the standard logic of organic synthesis, traditionally confined to the orchestration of transformations of functional groups ${ }^{1-4}$. Research efforts in targeting $\mathrm{C}-\mathrm{H}$ bond functionalization have mainly focused on the use of directing groups, present or pre-installed, or through the undirected activation of particularly reactive $\mathrm{C}-\mathrm{H}$ bonds. Extending this strategy to undirected $\mathrm{C}-\mathrm{H}$ bond functionalization requires the discovery of new strategies that would accurately activate and differentiate energetically akin C-H bonds, featured both in the substrate and in the product without cross-activation (Fig. 1a) ${ }^{5}$. This selective functionalization of alkanes, that do not possess directing groups, historically stands as one of the most challenging transformation in chemistry, particularly for undirected functionalization of strong and less electron-rich primary $\mathrm{C}-\mathrm{H}$ bonds ${ }^{6}$. Extensive efforts have been fueled by the prospect of rapidly valorizing this cheap and readily available organic feedstock, and few pioneering results have been achieved in alkane activation of primary $\mathrm{C}-\mathrm{H}$ bonds, most notably using homogeneous transition-metal based systems (Fig. 1b) ${ }^{7}$. Hartwig pioneered the first Rh-catalyzed regioselective terminal borylation with bispinacolatodiboron using alkanes in large excess ${ }^{8}$, and more recently the Ir-catalyzed activation of primary $\mathrm{C}-\mathrm{H}$ bonds using linear alkanes as limiting reagent ${ }^{9}$. An impressive regio- and enantioselective Rhcatalyzed carbene insertion into primary $\mathrm{C}-\mathrm{H}$ bonds have recently been reported by Davis (Fig. 1 b $)^{10}$ whereas Nelson developed the intermolecular non-selective $\mathrm{C}-\mathrm{H}$ insertion reaction of vinyl cations ${ }^{11,12}$. A recent very elegant report by Aggarwal on photoinduced electron transfer opened new doors to the $C\left(\mathrm{sp}^{3}\right)$ $\mathrm{H}$ borylation of alkanes although as a mixture of isomers in moderate yields ${ }^{13}$. Among indirect approaches, Martin and Baudoin have independently reported the unselective bromination of linear alkanes followed by subsequent and selective catalytic remote terminal carboxylation ${ }^{14}$ and arylation ${ }^{15}$ reactions, respectively whereas Huang converted alkanes into primary alkylsilanes through an initial dehydrogenation reaction using the initial alkanes as solvent ${ }^{16}$. Although these few selected examples highlight remarkable accomplishments for selective alkane functionalization, an inherent limitation of 
these strategies is that each transformation leads to a single type of functionalization and required, at the exception of the recent Ir-based strategy ${ }^{9}$, the alkanes as solvent or in very large excess. A general, highyielding and fully selective approach to the overall activation of primary $\mathrm{C}-\mathrm{H}$ bonds allowing a large number of different terminal functionalization $\left(\mathrm{FG}=\mathrm{I}, \mathrm{Br}, \mathrm{Cl}, \mathrm{SiR}_{3}, \mathrm{Bpin}, \mathrm{OH}, \mathrm{NR} \mathrm{R}_{2}, \mathrm{CN}, \mathrm{sp}^{3}\right.$ or $\mathrm{sp}^{2} \mathrm{C}-\mathrm{C}$ bond, etc) at the exclusive terminus position of an alkane, present in a stoichiometric amount, remains completely elusive.

Figure 1. Different strategies for the selective functionalization of alkanes. a, Existing challenges for the selective activation of $\mathrm{C}-\mathrm{H}$ bonds of linear hydrocarbons. $\mathbf{b}$, Homogeneous transition-metals allowing either selective primary $\mathrm{C}-\mathrm{H}$ activation reactions or unselective bromination or alkenylation followed by selective and catalytic remote terminal carboxylation, arylation and silylation reactions. c, Alternative strategy combining a dehydrogenation followed by a remote hydro-functionalization reaction. BDE = Bond dissociation energy.

We set out to develop this missing link by proposing an alternative approach that would first transform a stoichiometric amount of linear alkane into alkene, that would subsequently be engaged in a remote functionalization ${ }^{17,18}$ through a metal-catalyzed hydrometalation/migration sequence towards the formation of a primary organometallic species. The latter would subsequently react with a large variety of electrophiles providing a general access to the expected functionalized alkanes exclusively at the primary position (Fig. 1c). The activation of hydrocarbons through "dehydrogenation" is however, also a rather difficult transformation and heterogeneous ${ }^{19}$ or homogeneous-catalyzed reactions ${ }^{20}$ required high temperatures, inevitably leading to potential side reactions. In light of these inherent flaws of existing synthetic reagents for "dehydrogenation" reactions of alkanes ${ }^{21}$, we decisively turned our attention towards biocatalytic desaturase systems ${ }^{22}$, acknowledging the enzymatic ability to accurately distinguish even closely structurally related organic compounds and the potential of biocatalysis for important applications for sustainable processes ${ }^{23}$. The desaturation of non-activated C-C bonds have been mainly restricted to saturated fatty acids with the help of fatty acid desaturases (FADs). Most FADs do not act on the free fatty acid directly but rather on the corresponding thioester through an acyl carrier protein, practically limiting the applications only to carboxylic acids ${ }^{24,25}$. The selective and efficient desaturation of linear saturated unfunctionalized hydrocarbons into their corresponding unsaturated products was missing as alkenes were never directly assessed as catabolic intermediates in alkane biodegradation. Crucially, a mutant strain of Rhodococcus (Rhodococcus KSM-B-3M) ${ }^{26-28}$, obtained through random mutagenesis of the wild type strain Rhodococcus KSM-B-3, could hyperproduce internal alkenes from linear alkanes in rather low yields. We were therefore interested to implement this original low-yielding transformation as a general approach and evaluate whether synthetically useful yields of alkenes could be obtained. Following a thorough study of the reaction carried by the mutant (see Table $S 1$ for all details, Supplementary Materials), a remarkable monodehydrogenation transformation of alkane could be achieved by Rhodoccoccus KSM-B-3M ( $1 \mathrm{~g}$ wet cells) cultured in the presence of phosphate buffer $\left(\mathrm{K}_{2} \mathrm{HPO}_{4} / \mathrm{KH}_{2} \mathrm{PO}_{4}, 0.8 \mathrm{M}, \mathrm{pH}=6.4\right)$, monosodium glutamate (1.67 equiv.), thiamine $\cdot \mathrm{HCl}$ (3 mol\%) and magnesium sulfate $(2.8 \mathrm{~mol} \%)$ under ambient conditions $\left(30^{\circ} \mathrm{C}\right.$, aqueous solution, 
atmospheric pressure). Using this protocol, we were delighted to observe the transformation of hexadecane (1) into two major cis-hexadecene regioisomers $(2,80 \%$ cis-7-hexadecene $+20 \%$ cis-8hexadecene) with a remarkable $61 \%$ isolated yield based on the original alkane with a $83 \%$ dehydrogenation efficiency (alkenes/alkane) of aerobic incubation (Figure 2a). Several control experiments revealed the importance of the presence and concentration of all the reactants in solution to reach maximal olefin productivity (see Supplementary Materials). The biological material could also easily be recovered and reutilized into another batch of transformation for 4 consecutive runs further asserting the resiliency of this strain under the set conditions.

Figure 2. Scope of the Rhodococcus KSM-B-3M for regioselective desaturation of alkanes and other alkyl derivatives. a, The described protocol was optimized based on the conversion of $n$-hexadecane and recycling experiments. b, Scope of the range of desaturated linear alkanes. c, Scope of other desaturated xenobiotics using similar fermentation conditions. Reported yields were determined from the mass of organic material recovered after purification. Desaturation ratios were determined by GC and ${ }^{1} \mathrm{H}$ NMR. rpm = rotation per minute.

The fermentation reaction could be scaled-up to reach multigram scale synthesis, demonstrating the practicality of this procedure. The same protocol allowed to specifically desaturate linear $\mathrm{C}_{14}-\mathrm{C}_{20}$ alkanes (Figure $2 \mathbf{b}, 3-7$ ), reaching optimal conversion ratios for $n$-hexadecane and $n$-heptadecane. In all cases, the olefins produced were exclusively of (Z)-stereochemistry. Importantly, this microorganism enabled to mediate the desaturation of aliphatic xenobiotics such as terminally functionalized aliphatic derivatives (Figure 2c, for the entire scope, see Supplementary Materials). Interestingly, the formed olefins were accumulated over time, indicating that they were not further metabolized by the strain, underlying that linear a-olefins such as 1-hexadecene or 1-octadecene could undergo the biocatalytic desaturation to provide the corresponding unconjugated dienes in decent isolated yields $(\mathbf{8}, \mathbf{9})$. The reaction similarly proceeded with $n$-hexadecyl methyl ether, or chloro and fluoro hexa- and octadecane affording $a-$ functionalized Z-olefins (10-14) with somewhat variable yields and dehydrogenation ratios. These last examples place emphasis on the opportunity of chemoselectively desaturating aliphatic chains, even in the presence of potentially sensitive functional groups (8-14). This strongly contrasts with the relatively low functional group tolerance of synthetic reagents or catalysts capable of activating $\mathrm{C}-\mathrm{H}$ bonds in alkanes.

To shed some light on the biochemical pathways underlying the bacteria's ability to convert alkanes into alkenes, we first compared KSM B-3M with closely related Rhodococcus strains. Phylogenetic analysis revealed that Rhodococcus strain sp. 008 was the genetically available closest known strain to KSM B3M (Fig. 3a). In sharp contrast to KSM B-3M, when the strain sp. 008 was grown on hexadecane as a carbon source, it doubled its biomass in a 21 days experiment while consuming the alkane without any formation of alkenes (Fig. S1). These results suggest that sp. 008 uses hexadecane more efficiently than KSM B-3M through different metabolic pathways alluding that the mutation of the strain KSM B-3M potentially blocked subsequent alkane metabolism pathways ${ }^{27}$. To further identify the genes responsible for this transformation, we performed transcriptomic comparison between KSM B-3M and sp. 008, both 
growing independently on hexadecane and dodecane as carbon sources. Principal Component Analysis (PCA) of the transcriptome revealed that strain differences explain $97 \%$ of the variability in gene expression (PC1, Figure $3 \mathbf{b}$ ). Only $1 \%$ of the variation is attributed to the difference in the carbon source observed in KSM B-3M but not in sp. 008 (PC2, Figure 3b). This result indicates distinct cell metabolism pathways amongst the two strains regarding alkane metabolism. Differential gene expression analysis comparing the transcriptome of the two strains on hexadecane revealed 1331 genes with at least a tenfold excess (adjusted p-value $<0.05$, FDR). Interestingly, 1283 of these genes were higher in sp. 008 while only 48 genes were higher in KSM B-3M, indicating a specific metabolic activity that defines KSM B-3M dehydrogenation ability. Strikingly, the most abundant gene in KSM B-3M, 250 times higher than in sp. 008, was found to be acyl-CoA desaturase (Fig. 3c).

Figure 3. a, Phylogenetic tree of selected Rhodococcus strains. As shown sp. 008 is the closest strain to KSM B-3M among the selected strains. b, Principal Component Analysis (PCA) of different transcriptomic plans in KSM B-3M and sp. 008. Evidently $97 \%$ of the variation is explained by PC1 which corresponds to differences between strains. Group 1: KSM sp. 008. Group 2: hexadecane dodecane c, High levels of Acyl-CoA desaturase expression and low levels of fatty acid degradation pathway expression in KSM as compared to sp. 008 when growing on hexadecane as carbon source. The volcano plot representing the significance of each gene versus its expression differential between KSM B-3M and sp. 008. Indicated in red genes that are: $\mathbf{c 1}$, overexpressed in KSM B-3M by at least $2^{5}$ fold. $\mathbf{c 2}$, Genes overexpressed in sp. 008 by at least $2^{10}$ fold. d, q-PCR targeting acyl-CoA desaturase, ferredoxin reductase and gene encodes hypothetical protein in sp. 008 vs. KSM-B-3M, on nutrient broth (NB) solution (24h) or hexadecane (7 days). Two biological repeats for each treatment.

Moreover, two genes adjacent to acyl-CoA desaturase on the KSM B-3M genome, ferredoxin reductase and a gene encoding for a hypothetical protein were also much highly expressed in KSM B-3M than in sp. 008 defining the region containing these three genes as a putative operon. Interestingly, acyl-CoA desaturase catalyzes the conversion of saturated fatty acids to unsaturated forms and ferredoxin reductase is an electron donor required for its function ${ }^{29}$. Quantitative-PCR (q-PCR) targeting the three genes of the operon, namely acyl-CoA desaturase, ferredoxin reductase and the hypothetical protein confirmed their high expression levels induced by hexadecane in KSM B-3M (Fig. 3d). Proteomic analysis demonstrated similar results at the proteins level. It was further subjected to enrichment analysis, which revealed four metabolic pathways that are higher in sp. 008 compared to KSM B-3M. The beta-oxidation pathway for fatty acid degradation is the most enriched among these pathways (adjusted $p$-value $=2.6 \mathrm{E}$ 4) with ten genes higher in sp. 008 (Table S2, Fig. S2). Combining the results from the transcriptomic and proteomic analyses of KSM B-3M indicate an over expression of acyl-CoA desaturase operon with a lack of subsequent beta oxidation pathways, indicating a regioselective dehydrogenation reaction of alkanes without subsequent b-oxidation. Based on these findings molecular docking was then performed in order to predict hexadecane binding to acyl-CoA desaturase. For that we used the 3D structure of a mouse Stearoyl-Coenzyme A Desaturase-1, the closest acyl-CoA desaturase homolog with PDB structure ${ }^{30,31}$. Despite a rather low $17 \%$ overall sequence identity, the binding pocket residues showed higher than $30 \%$ 
pairwise identity and 30\% similarity identity between mouse and Rhodococcus meaning that both proteins share very similar desaturase functions (Table S3, Fig. S3) ${ }^{32}$. Based on our observation that the binding pocket is much more conserved than the overall fold, we generated a chimeric Rhodococcusmouse binding site 3D structure in silico and proposed a model for the binding pocket that presents a highly hydrophobic lower half, with eight AA (two conserved Trp, four Val, one Leu and one Tyr), (Fig. 4a) and a more polar and charged upper half. By exploring the minimal distance between various hydrocarbon chains and the $\mathrm{C} B$ atom of Val 257 as specific buried binding pocket (Fig. 4a and $4 \mathbf{b}$ ), hexadecane is at a short $7.0 \AA$ distance while it is at a longer distance for all other substrates that were not dehydrogenated in our reaction conditions.

Fig 4. a, Chimera Rhodococcus-mouse binding pocket used for the docking experiments with hexadecane docking shown in light green. All residues that do not belong to the binding pocket are showed as a white ribbon. Rhodococcus hydrophobic and aromatic residues are colored tan, polar and charged residues in light blue, $\mathrm{Zn}$ atoms in yellow, hydrogens in white and the ribbon backbone atoms (from mouse) in transparent white. $\mathbf{b}$, Best LeDock docked for hexadecane, hexadecane is colored in light green.

With a practical and powerful strategy in hand to access stoichiometrically mono-unsaturated hydrocarbon derivatives using strain KSM B-3M, we proceeded by examining the next step of our approach, namely the remote and selective terminal functionalization reaction ${ }^{33}$. This strategy capitalizes on the remarkable ability of transition-metal complexes to promote rapid and long-range migration along linear hydrocarbon chains under specific conditions ${ }^{34}$. This equilibrium is usually shifted towards the predominant formation of the anti-Markovnikov terminal organometallic intermediate, enabling eventual terminal derivatization either through reductive elimination or subsequent electrophilic functionalization. If successful, an inherent advantage of our strategy is that the chemical outcome is independent of the position of the initial double bond, thus serving as a regioconvergent remote activation of terminal $\mathrm{C}-\mathrm{H}$ bonds. To this purpose, we prospectively identified several transition-metal based catalysts and reagents capable of affecting such long-range functionalization upon our biodesaturated substrates (see Figure 5). As a proof of concept, we successfully engaged the mixture of hexadecenes produced from $n$ hexadecane through the biocatalytic desaturation process (Figure $5 \mathbf{a}$, green arrow) into various metalmediated remote hydrofunctionalization reactions (Figure $5 \mathbf{a}$, red arrow), thoroughly optimized to reach excellent site-selectivities (a>98:2 in all cases). For instance, the terminal alkylborane 15 could be selectively synthesized using pinacolborane and a catalytic mount of Co-based pincer complex at room temperature ${ }^{35}$. Furthermore, trichloroalkylsilanes - which are essential components to polymer and material sciences- could be accessed from 2 and trichlorosilane using $5 \mathrm{~mol} \%$ of chloroplatinic acid $\left(\mathrm{H}_{2} \mathrm{PtCl}_{6} \cdot 6 \mathrm{H}_{2} \mathrm{O}\right)$ as catalyst ${ }^{36}$. In a one-pot operation, subsequently treating the resulting trichlorohexadecylsilane with different Grignard reagents (i.e. $\mathrm{MeMgBr}$ and vinylMgBr) enabled us to provide either trimethyl- or trivinyl-hexadecylsilane $\mathbf{1 6}$ and $\mathbf{1 7}$ in good overall yields. Hydrozirconation reactions have the advantage of enabling an even broader palette of electrophilic functionalizations ${ }^{37}$. We found that treating 2 with a mixture of $\mathrm{Cp}_{2} \mathrm{ZrCl}_{2}$ and Red-Al ${ }^{38}$ in THF at $40{ }^{\circ} \mathrm{C}$ for $18 \mathrm{~h}$ yielded the 
terminal hexadecylzirconocene chloride intermediate. Subsequent electrophilic trapping with $\mathrm{NCS}, \mathrm{Br}_{2}$ and $\mathrm{I}_{2}$ exclusively afforded the corresponding 1-halogenohexadecanes 18-20 in excellent overall yields. Interestingly, treatment of the same organozirconocene intermediate with $t \mathrm{BuOOH}$ yielded cetyl alcohol (21) while addition of $\mathrm{H}_{2} \mathrm{NOSO}_{3} \mathrm{H}$ and $\mathrm{MeNHOSO}_{3} \mathrm{H}^{39}$ afforded 1-hexadecylamine and $\mathrm{N}$-methyl hexadecylamine, respectively (22 and 23 ). Alternatively, addition of TMSCN and $\mathrm{I}_{2}$ provided $n-$ heptadecanonitrile $\mathbf{2 4}$ in $\mathbf{7 5 \%}$ yield. Importantly, C-C bond formation could also be achieved in the presence of a catalytic amount of $\mathrm{Cu}(\mathrm{I})$ salts to provide either the allenylated or allylated products 25-27, respectively. Regarding other $\mathrm{sp}^{3}-\mathrm{sp}^{3}$ cross-coupling reactions, we implemented the nickel-based catalytic system ${ }^{40}$ to couple the bioproduced hexadecenes with an alkyl iodide to exclusively provide linear product 28 .

Figure 5. Illustration of the large array of derivatives accessible from different remote hydrofunctionalization procedures applied to: a, the mixture of cis-hexadecenes 2 , bioproduced from $n$ hexadecane, and b, 1-methoxyhexadecenes, bioproduced from 1-methoxyhexadecane. Yields were either evaluated by GC (with internal standard in parentheses) or after purification by column chromatrography on silica gel. All site-selectivities in 15-28 were above 98:2 (evaluated by NMR).

Finally, our approach could also target the directionally selective and remote C-O bond activation as illustrated with a mixture of 1-methoxyhexadecenes, bioproduced from 1-methoxyhexadecane (Figure $5 b)$. In this case, the ruthenium-catalyzed olefin migration was thermodynamically guided by the formation of the corresponding enol ether, which is then cross-coupled with $\mathrm{PhMgBr}$ in the presence of catalytic amount of $\left[\mathrm{NiCl}_{2}\left(\mathrm{PPh}_{3}\right)_{2}\right]$, yielding $b$-pentadecylstyrene 29 in a one-pot sequence ${ }^{41,42}$.

In summary, we herein report an efficient, unified and general two-step strategy to selectively functionalize terminal $\mathrm{C}-\mathrm{H}$ bonds of linear alkanes, yielding a large variety of functionalized alkyl derivatives $(\mathrm{FG}=\mathrm{I}, \mathrm{Br}$, $\mathrm{Cl}, \mathrm{SiR}_{3}, \mathrm{Bpin}, \mathrm{OH}, \mathrm{NR}_{2}, \mathrm{CN}, \mathrm{sp}^{3}$ or sp${ }^{2} \mathrm{C}-\mathrm{C}$ bond, etc) while avoiding the use of linear $\alpha$-olefins. The originality of this approach stems from the combined use of bacterial alkane activation with the metalmediated remote hydro-functionalization, which remarkably prevents side-reactions, reaching synthetically useful yields of linear alkyl derivatives. We believe these findings will inspire the design and development of even more efficient (semi-) synthetic strategies targeting the highly desirable dehydrogenation or other selective functionalization of hydrocarbon chains.

\section{Declarations}

Acknowledgments: This research was supported by the European Research Council under the Excellence Science H2020 Program of the European Community (ERC grant agreement $n^{\circ} 786976$ ) and by Russell Berrie Nanotechnology Institute, Technion. The National Institute of Technology and Evaluation (NITE, Japan) is acknowledged for furnishing samples of KSM-3-BM under the code FERM BP-1531. YZ was supported by the Planning and Budgeting Committee of the Council for Higher Education of Israel. The 
Technion Genome Center and The Smoler Proteomics Center at the Technion are acknowledged for whole-genome \& transcriptome sequencing and for the proteomic analysis.

Author contributions: JB, AC, MC, YZ, G-MH, IM and IM planned, conducted and analyzed the chemical experiments. KB-R, JB, IK, NE, HH-V and YK planned, conducted and analyzed the biological experiments. FG, KB-R and GH conducted the genomic and proteomic analysis as well as docking experiments. IM conceived and directed the project and wrote the manuscript with contributions by JB, KB-R, AC, GH, FG and YK. All authors contributed to discussions. Correspondence and requests for materials should be addressed to IM;

Competing interests: JB, KBR, IK, YK, MC and IM* declare competing financial interests.

Data and materials availability: All data is available in the main text or the supplementary materials. All data, code, and materials used in the analysis are available in some form to any researcher for purposes of reproducing or extending the analysis.

\section{References}

1. Hartwig, J. F. \& Larsen, M. A. Undirected, Homogeneous C-H Bond Functionalization: Challenges and Opportunities. ACS Cent. Sci. 2, 281-292 (2016).

2. Hartwig, J. F. Evolution of C-H Bond Functionalization from Methane to Methodology. Am. Chem. Soc. 138, 2-24 (2016).

3. Brückl, T., Baxter, R. D., Ishihara, Y. \& Baran, P. S. Innate and Guided C-H Functionalization Logic. Acc. Chem. Res. 45, 826-839 (2012).

4. Sames, D. \& Godula, K. C-H Bond Functionalization in Complex Organic Synthesis. Science 312, 67$72(2006)$.

5. Hartwig, J. F. Catalyst-Controlled Site-Selective Bond Activation. Chem. Res. 50, 549-555 (2017).

6. Goldberg, K. I. \& Goldman, A. S. Large-Scale Selective Functionalization of Alkanes. Chem. Res. 50, 620-626 (2017).

7. Pérez, P. J. Alkane C-H activation by single-site metal catalysis, (Springer, 2012).

8. Chen, H., Sclecht, S., Semple, T. C. \& Hartwig, J. F. Thermal, Catalytic, Regiospecific Functionalization of Alkanes. Science 287, 1995-1997 (2000).

9. Oeschger, R., Su, B., Yu, I., Ehinger, C., Romero, E., He, S. \& Hartwig, J. F. Diverse Functionalization of Strong Alkyl C-H Bonds by Undirected Borylation. Science 368, 736-741 (2020).

10. Liao, Y. K., Yang, Y.-F., Li, Y., Sanders, J. N., Houk, K. N., Musaev, D. C. \& Davies, H. M. L. Design of Catalysts for Site-Selective and Enantioselective Functionalization of Non-Activated Primary C-H Bonds. Nature Chem. 10, 1048-1055 (2018).

11. Popov, S., Shao, B., Bagdasarian, A. L., Benton, T. R., Zou, L., Yang, Z., Houk, K. N. \& Nelson, H. M. Teaching an Old Carbocation New Tricks: Intermolecular C-H Insertion Reactions of Vinyl Cations. 
Science 361, 381-387 (2018).

12. Shao, B., Bagdasarian, A. L., Popov. S. \& Nelson, H. M. Arylation of Hydrocarbons Enabled by Organosilicon Reagents and Weakly Coordinating Anion. Science 355, 1403-1407 (2017).

13. Shu, C., Noble, A. \& Aggarwal, V. K. Metal-free photoinduced C( $\left(\mathrm{sp}^{3}\right)$-H borylation of alkanes. Nature, 586, 714-720 (2020).

14. Juliá-Hernández, F., Moragas, T., Cornella, J. \& Martin, R. Remote Carboxylation of Halogenated Aliphatic Hydrocarbons with Carbon Dioxide. Nature 545, 84-88 (2017).

15. Dupuy, S., Zhang, K. F., Goutierre, A.-S. \& Baudoin, O. Terminal-Selective Functionalization of Alkyl Chains by Regioconvergent Cross-Coupling. Chem. Int. Ed. 55, 14793-14797 (2016).

16. Jia, X. \& Huang, Z. Conversion of Alkanes to Linear Alkylsilanes Using an Iridium-Iron-Catalysed Tandem Dehydrogenation-Isomerization-Hydrosilylation. Nature Chem. 8, 157-161 (2016).

17. Vasseur, A., Bruffaerts, J. \& Marek, I. Remote Functionalization Through Alkene Isomerization. Nature Chem. 8, 209-219 (2016).

18. Sommer, H., Julia-Hernandez, F., Martin, R. \& Marek, I. Walking Metals for Remote Functionalization. ACS Cent. Sci. 4, 153-165 (2018).

19. Sattler, J. J. H. B., Ruiz-Martinez, J., Santillan-Jimenez, E. \& Weckhuysen, B. M. Catalytic Dehydrogenation of Light Alkanes on Metals and Metal Oxides. Rev. 114, 10613-10653 (2014).

20. Kumar, A., Bhatti, T. M., \& Goldman, A. S. Dehydrogenation of Alkanes and Aliphatic Groups by Pincer-Ligated Metal Complexes. Rev. 117, 12357-12384 (2017).

21. West, J. G. \& Sorensen, E. J. Development of a Bio-Inspired Dual Catalytic System for Alkane Dehydrogenation. J. Chem. 57, 259-269 (2017).

22. Honig, M., Sondermann, P., Turner, N. J. \& Carreira, E. M. Enantioselective Chemo- and Biocatalysis: Partners in Retrosynthesis. Chem. Int. Ed. 56, 8942-8973 (2017).

23. Lewis, J. C., Coelho, P. S. \& Arnold, F. H. Enzymatic Functionalization of Carbon-Hydrogen Bonds. Soc. Rev. 40, 2003-2021 (2011).

24. Qu, G., Li, A., Acevedo-Rocha, C. G., Sun, Z. \& Reetz, M. T. The crucial role of methodology development in directed evolution of selective enzymes. Chem. Int. Ed. 59, 13204-23231 (2020).

25. Ayala, M. \& Torres, E. Enzymatic Activation of Alkanes: Constraints and Prospective. Catal., A: General 272, 1-13 (2004).

26. Koike, K., Takaiwa, M., Kimura, Y., Inoue, S. \& Ito, S. Substrate Specificity of Regiospecific Desaturation of Aliphatic Compounds by a Mutant Rhodococcus Biosci. Biotechnol. Biochem. 64, 1064-1066 (2000).

27. Koike, K. et al. Regiospecific Internal Desaturation of Aliphatic Compounds by a MutantRhodococcus Appl. Environ. Microbiol. 65, 5636-5638 (1999).

28. Takeuchi, K., Koike, K. \& Ito, S. Production of Cis-Unsaturated Hydrocarbons by a Strain of Rhodococcus in Repeated Batch Culture with a Phase-Inversion, Hollow-Fiber System. Biotechnol. 14, 179-186 (1990). 
29. Dar A. A. et al. TheFAD2 Gene in Plants: Occurrence, Regulation, and Role. Plant. Sci. doi: 10.3389/fpls.2017.01789, (2017).

30. Berman H. M. et al., The Protein Data Bank Nucleic Acids Research, 28, 235-242 (2000).

31. Bai Y. et al., X-Ray Structure Of A Mammalian Stearoyl-Coa Desaturase. Nature 524, 252-256 (2015).

32. Soding, J., Biegert, A. \& Lupas, A. N. The HHpred Interactive Server for Protein Homology Detection and Structure Prediction. Nucleic Acids Research, 33, W244-W248 (2005).

33. Singh, S., Bruffaerts, J., Vasseur, A, \& Marek, I. A Unique Pd-Catalysed Heck Arylation as a Remote Trigger for Cyclopropane Selective Ring-Opening. Nature Comm. 8, 14200 (2017).

34. Bruffaerts, J., Pierrot, D. \& Marek, I. EfficientaAnd Stereodivergent Synthesis of Unsaturated Acyclic Fragments Bearing Contiguous Stereogenic Elements. Nature Chem. 10, 1164-1170 (2018).

35. Obligacion, J. V. \& Chirik, P. J. Bis(imino)pyridine cobalt-catalyzed alkene isomerization hydroboration: A strategy for remote hydrofunctionalization with terminal selectivity. Am. Chem. Soc. 135, 19107-19110 (2013).

36. Saam, J. C. \& Speier, J. L. The Addition of Silicon Hydrides to Olefinic Double Bonds. The addition to Non-Terminal Olefins in the presence of Chloroplatinic Acid. Am. Chem. Soc. 80, 4104-4106 (1958).

37. Masarwa, A. et al., Merging Allylic Carbon-Hydrogen and Selective Carbon-Carbon Bond Activation. Nature 505, 199-203 (2014).

38. Gibson, T. \& Tulich, Novel Synthesis oof Long-Chain Alkyl Compounds. J. Org. Chem. 46, 1821-1823 (1981).

39. Strom, A. E. \& Hartwig, J. F. One-Pot Anti-Markovnikov Hydroamination of Unactivated Alkenes by Hydrozirconation and Amination. Org. Chem. 78, 8909-8914 (2013).

40. Zhou, F., Zhu, J., Zhang, Y. \& Zhu, S. NiH-Catalyzed Reductive Relay Hydroalkylation: A Strategy for the Remote $\mathrm{C}\left(\mathrm{sp}^{3}\right)-\mathrm{H}$ Alkylation of Alkenes. Chem. Int. Ed. 57, 4058-4062 (2018).

41. Ho, G.-H., Judkele, L., Bruffaerts, J. \& Marek, I. Metal-Catalyzed Remote Functionalization of $\omega$-Ene Unsaturated Ethers: Towards Functionalized Vinyl Species. Chem. Int. Ed. 57, 8012-8016 (2018).

42. Romano, C. \& Mazet, C. Multicatalytic Stereoselective Synthesis of Highly Substituted Alkenes by Sequential Isomerization/Cross-Coupling Reactions. Am. Chem. Soc. 140, 4743-4750 (2018).

\section{Figures}


a, Undrected CH Funcionalization
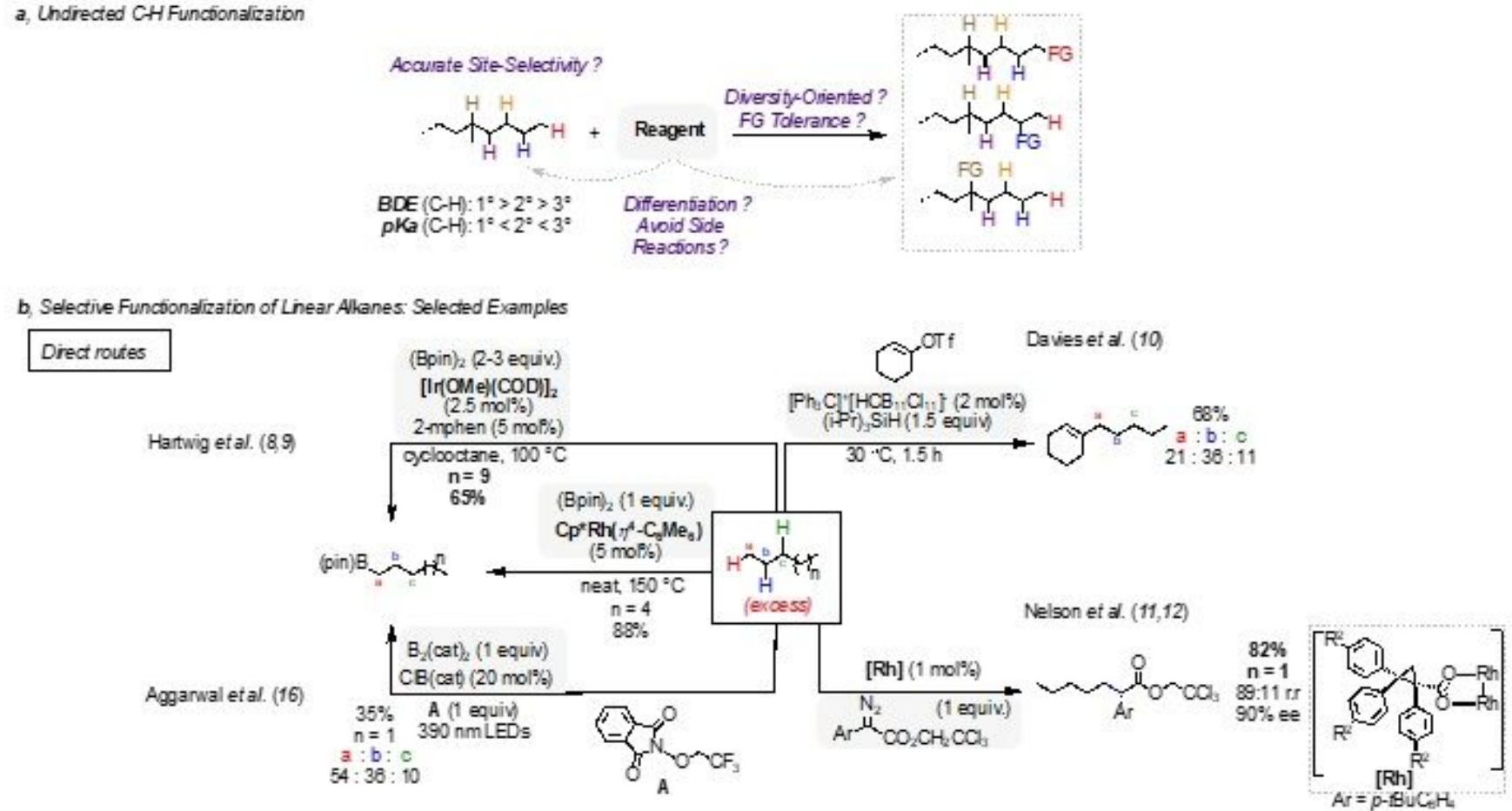

Indrect routes

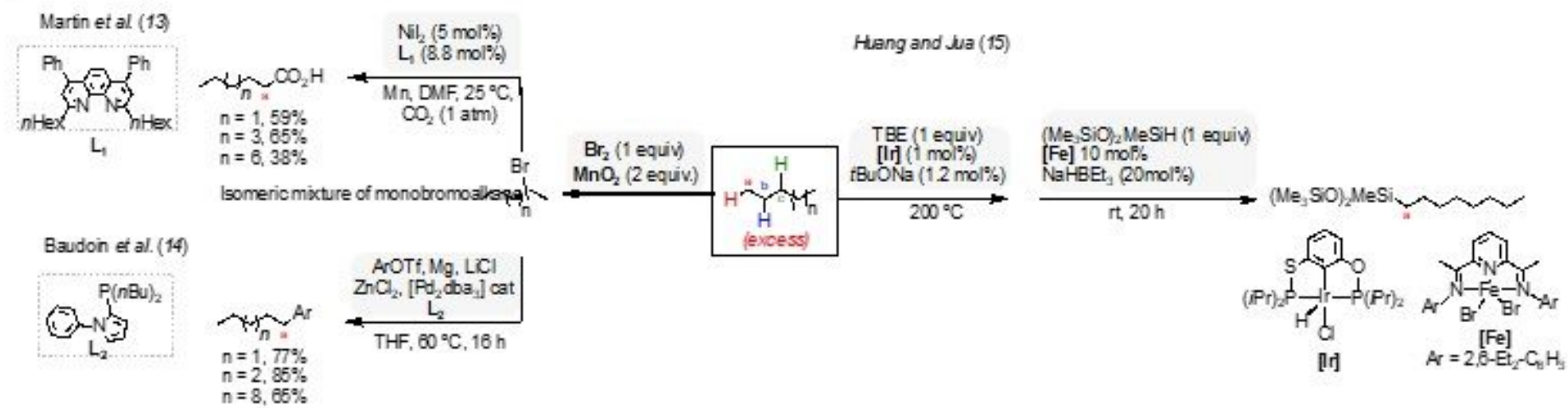

c, Ou Approach

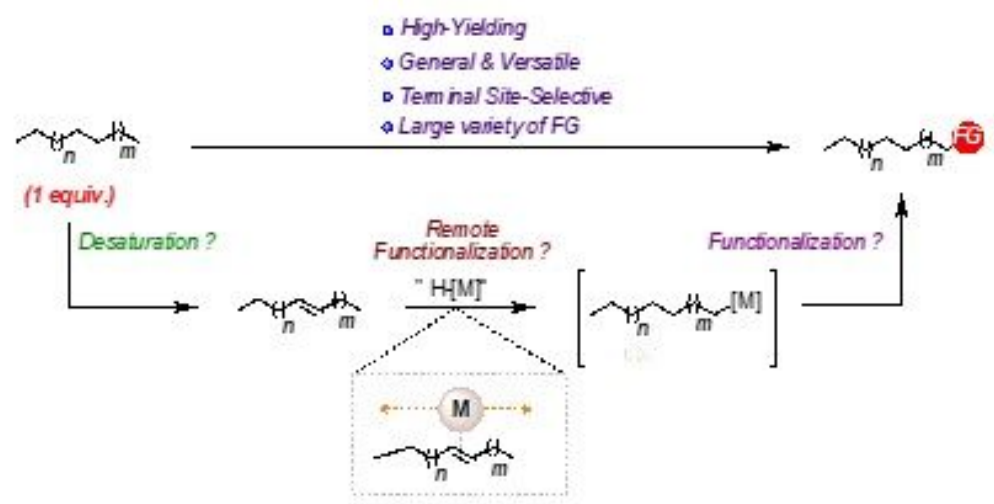

Figure 1

Different strategies for the selective functionalization of alkanes. a, Existing challenges for the selective activation of $\mathrm{C}-\mathrm{H}$ bonds of linear hydrocarbons. $\mathrm{b}$, Homogeneous transition-metals allowing either selective primary $\mathrm{C}-\mathrm{H}$ activation reactions or unselective bromination or alkenylation followed by selective and catalytic remote terminal carboxylation, arylation and silylation reactions. c, Alternative strategy 
combining a dehydrogenation followed by a remote hydro-functionalization reaction. BDE = Bond dissociation energy.

a,

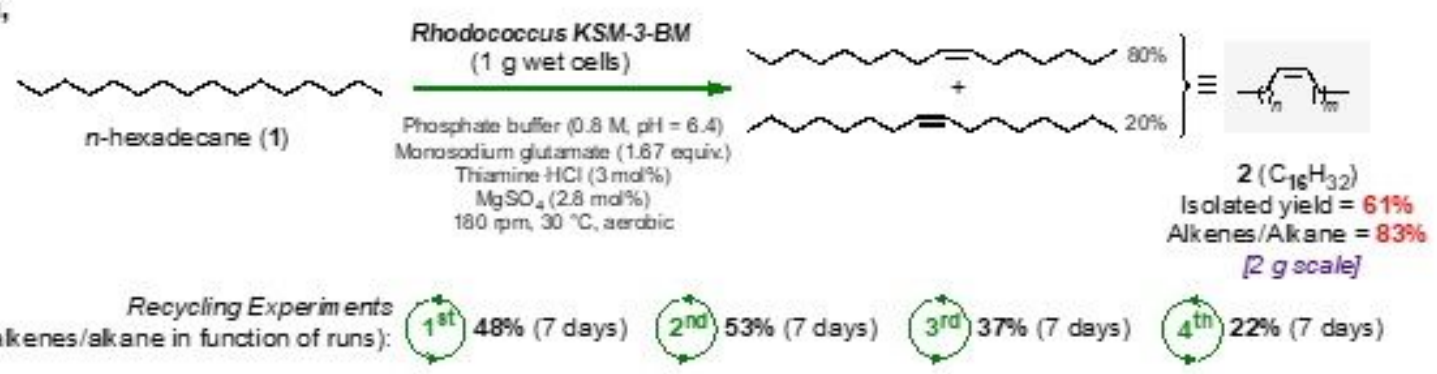

b,

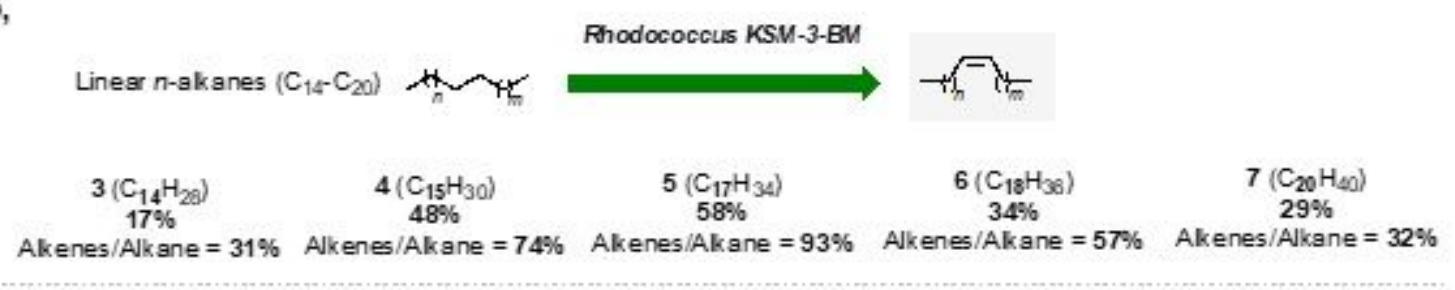

c,

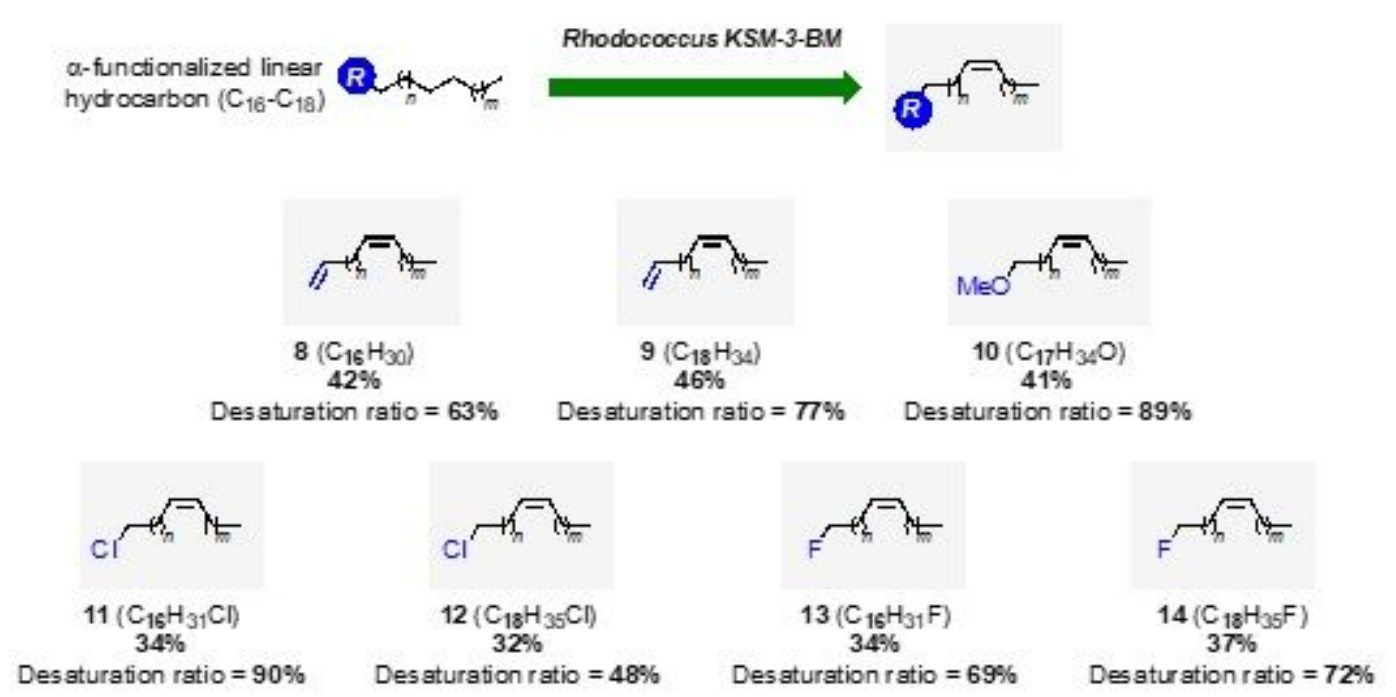

\section{Figure 2}

Scope of the Rhodococcus KSM-B-3M for regioselective desaturation of alkanes and other alkyl derivatives. a, The described protocol was optimized based on the conversion of n-hexadecane and recycling experiments. b, Scope of the range of desaturated linear alkanes. c, Scope of other desaturated xenobiotics using similar fermentation conditions. Reported yields were determined from the mass of organic material recovered after purification. Desaturation ratios were determined by GC and 1H NMR. rpm = rotation per minute. 


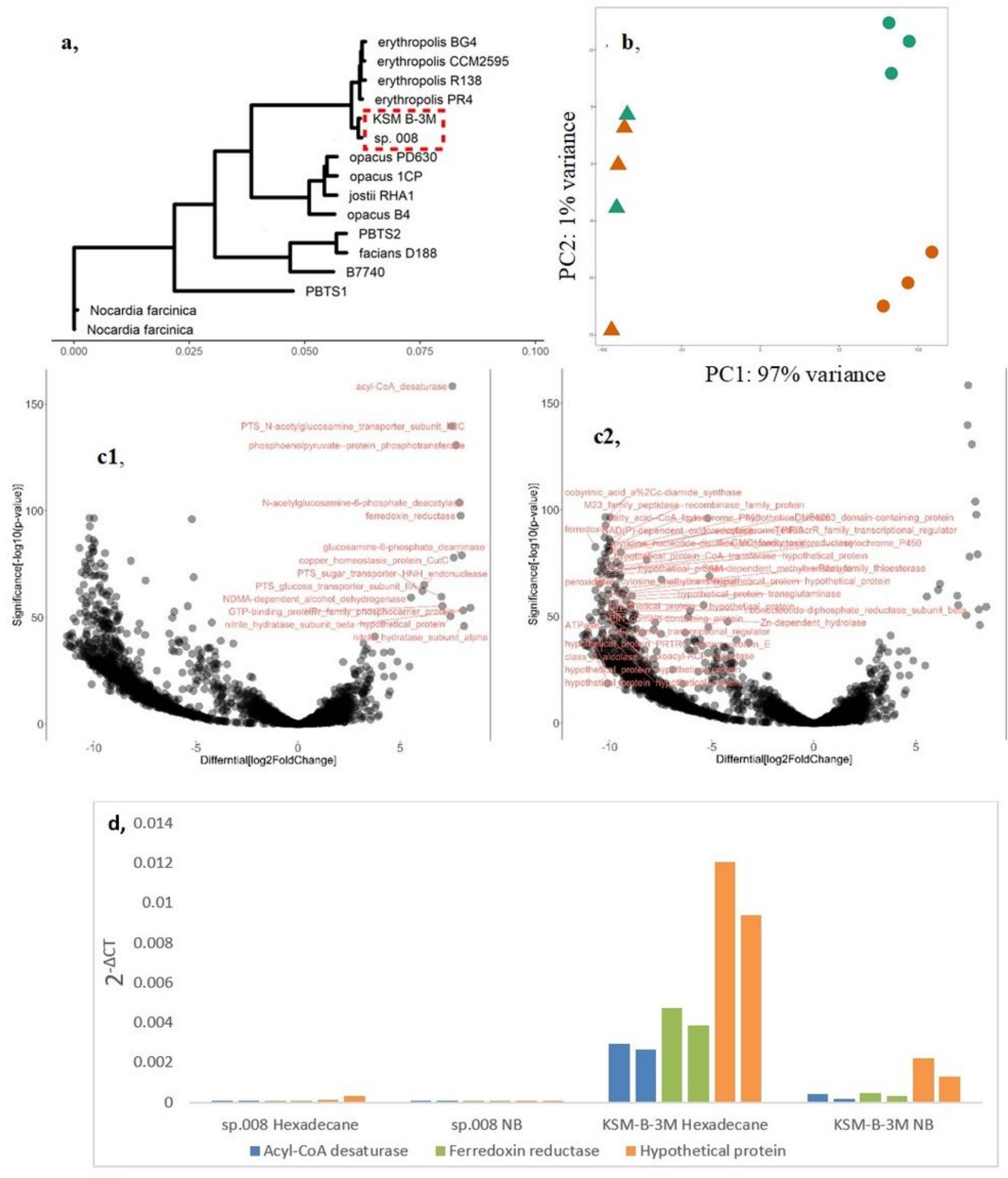

\section{Figure 3}

a, Phylogenetic tree of selected Rhodococcus strains. As shown sp. 008 is the closest strain to KSM B-3M among the selected strains. b, Principal Component Analysis (PCA) of different transcriptomic plans in KSM B-3M and sp. 008. Evidently $97 \%$ of the variation is explained by PC1 which corresponds to differences between strains. Group 1: KSM sp. 008. Group 2: hexadecane dodecane c, High levels of Acyl-CoA desaturase expression and low levels of fatty acid degradation pathway expression in KSM as 
compared to sp. 008 when growing on hexadecane as carbon source. The volcano plot representing the significance of each gene versus its expression differential between KSM B-3M and sp. 008. Indicated in red genes that are: c1, overexpressed in KSM B-3M by at least 25 fold. c2, Genes overexpressed in sp. 008 by at least 210 fold. d, q-PCR targeting acyl-CoA desaturase, ferredoxin reductase and gene encodes hypothetical protein in sp. 008 vs. KSM-B-3M, on nutrient broth (NB) solution (24h) or hexadecane (7 days). Two biological repeats for each treatment.

a,

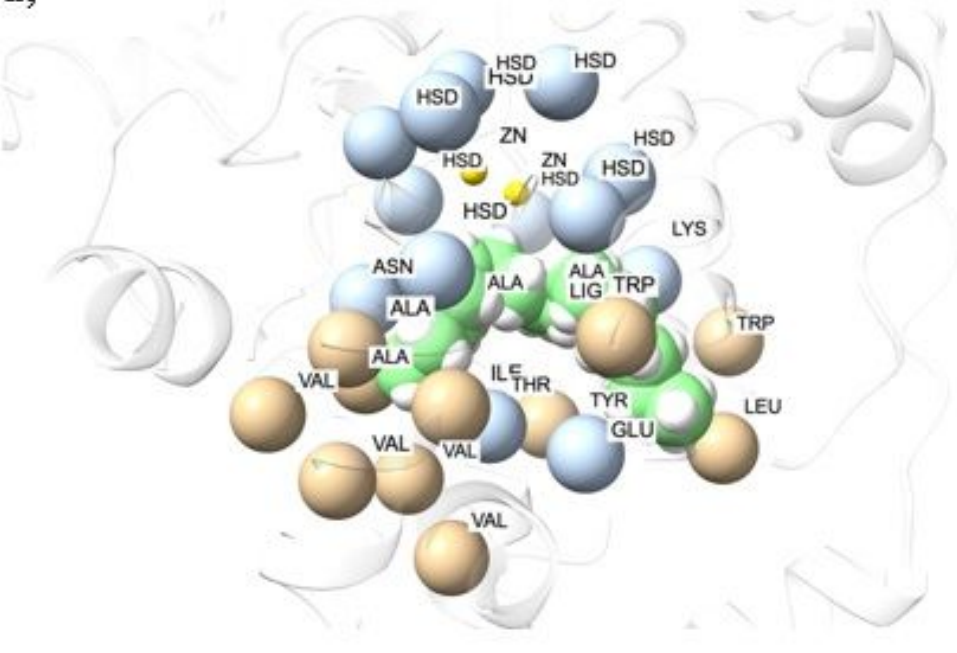

b,

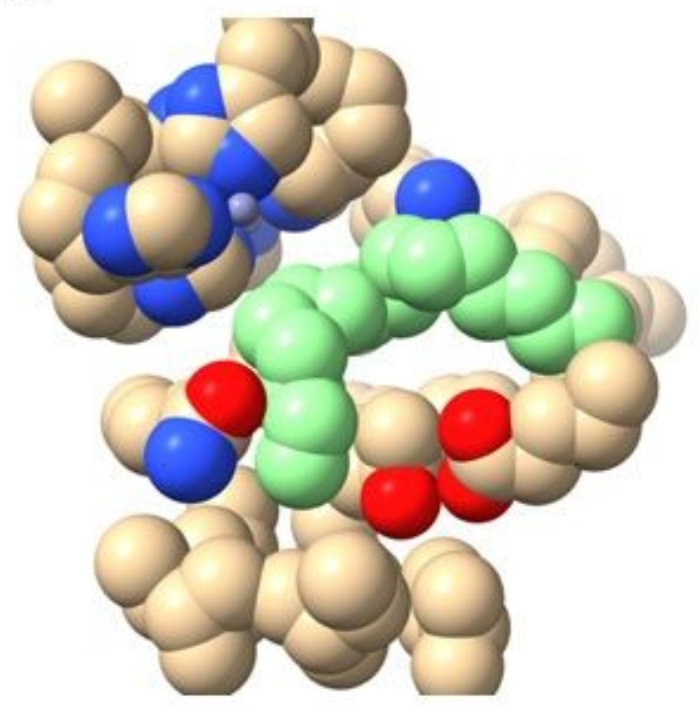

Figure 4

a, Chimera Rhodococcus-mouse binding pocket used for the docking experiments with hexadecane docking shown in light green. All residues that do not belong to the binding pocket are showed as a white ribbon. Rhodococcus hydrophobic and aromatic residues are colored tan, polar and charged residues in light blue, $\mathrm{Zn}$ atoms in yellow, hydrogens in white and the ribbon backbone atoms (from mouse) in transparent white. b, Best LeDock docked for hexadecane, hexadecane is colored in light green. 


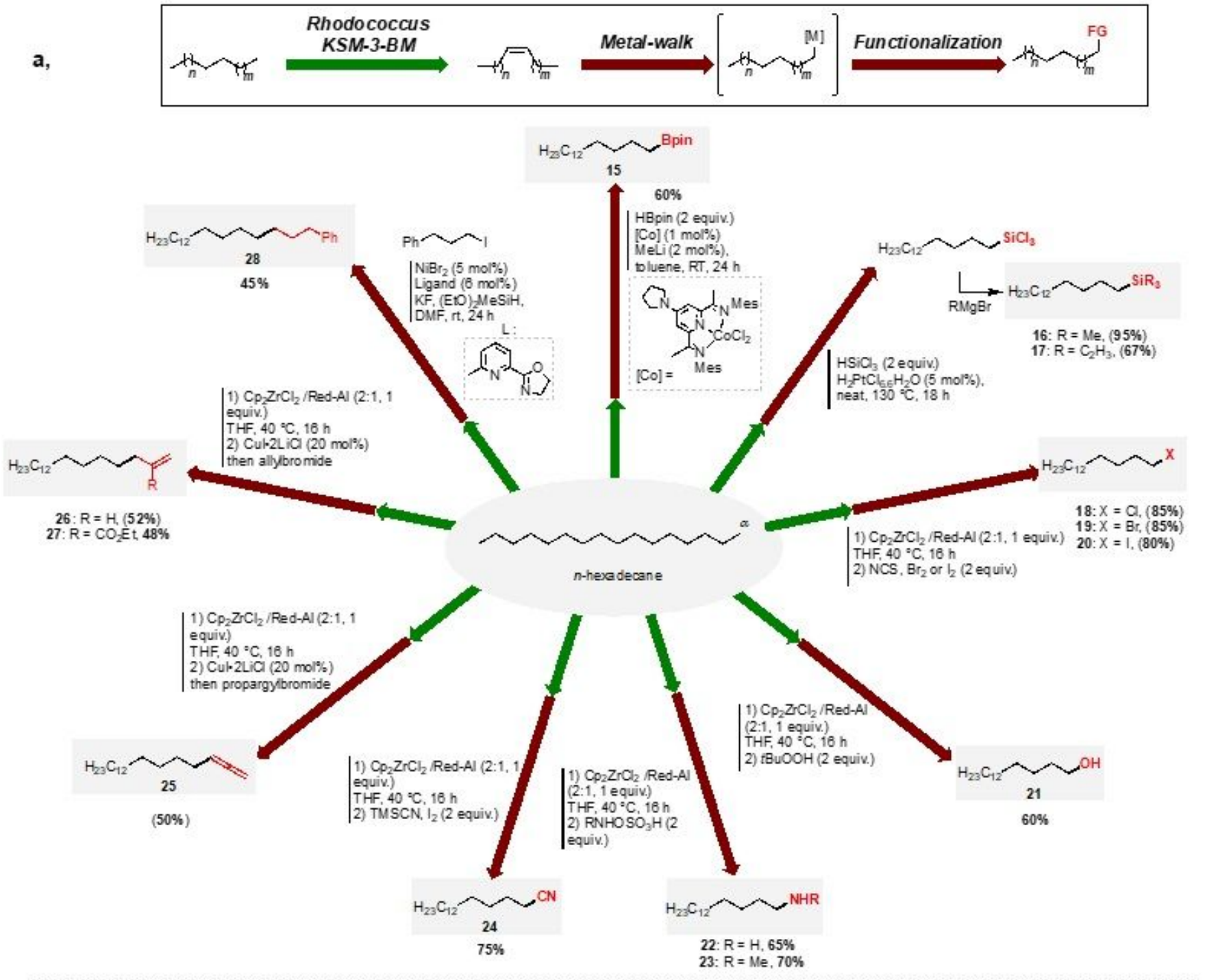

b,

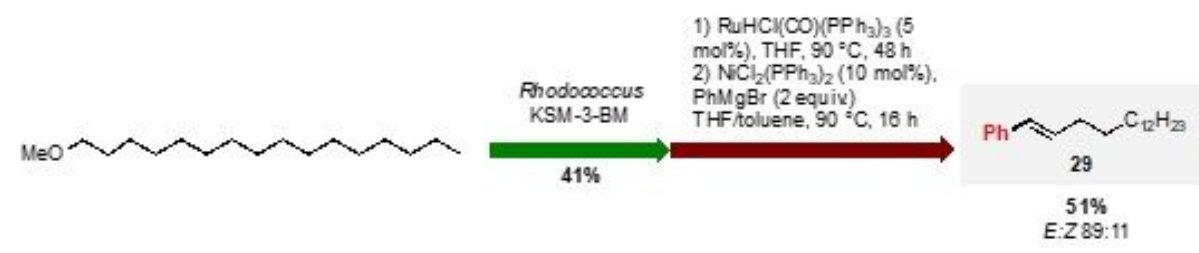

\section{Figure 5}

Illustration of the large array of derivatives accessible from different remote hydrofunctionalization procedures applied to: $a$, the mixture of cis-hexadecenes 2 , bioproduced from n-hexadecane, and b, 1methoxyhexadecenes, bioproduced from 1-methoxyhexadecane. Yields were either evaluated by GC (with internal standard in parentheses) or after purification by column chromatrography on silica gel. All siteselectivities in 15-28 were above 98:2 (evaluated by NMR).

\section{Supplementary Files}

This is a list of supplementary files associated with this preprint. Click to download. 
- NatureChemistrySupplementaryMaterials.docx 